\title{
Pricing Strategy Research of Chinese Video Website Platform in Internet Era
}

\author{
Yiran Wang ${ }^{1, *}$ \\ ${ }^{1}$ Soochow Foreign Language School, No. 188 YuCheng Rd Xiangcheng District Suzhou, Jiangsu, China \\ *Corresponding author. Email: wenyi.luo@bu.edu
}

\begin{abstract}
The network video platform has gradually occupied a large share in the field of television media. However, as a hot field in the Internet era, academic research on online video platforms is relatively scarce. Based on the characteristics of online video platforms, this paper studies the pricing strategies of iQIYI and Tencent Video. The profit model of the online video platforms is mature. It has developed from advertising profit in the initial stage to content profit at present, and various profit models coexist. The main analysis methods used in this paper are literature analysis method, case study and comparative analysis, finally concluded the development status and trend analysis of Chinese video website industry and current situation of pricing strategy of Chinese video website, discussing the problems of the pricing strategy and the optimization of pricing strategy for Chinese video websites. This paper creatively considers the pricing strategy of the online video platforms, obtains some reference conclusions for the actual operation decisions of platform enterprises, and provides a reference for the research of the online video platform field.
\end{abstract}

\section{Keywords: Internet Era, Pricing strategy, Chinese video website, Marketing}

\section{INTRODUCTION}

\subsection{Research background}

A video website is an online media platform for watching movies, dramas, and variety shows. However, in the early stage of Internet development, video platforms did not show great energy and vitality due to the backward technology of Internet hardware, network memory, and network bandwidth. As the first video Player software, Windows Media Player stimulated the enthusiasm of netizens and triggered the huge energy in the field of video. More and more users are participating in video platforms. As a result, video platforms as a new industry gradually began to enter the market.

In recent years, the market scale of China's video websites has continued to expand, and the number of users of major video websites continues to grow. To gain a larger market and attract more users in the increasingly fierce market competition environment, the pricing strategy of the platform plays a crucial role in the future development of video websites. The rapid development of the video website payment business is inseparable from the growing large user groups. Paying for video has become the norm in recent years, and it's gaining momentum. With the development of network technology, its operation mode is becoming more and more convenient, and even has reached the point of unlimited recharge at anytime and anywhere. The user pays consciousness is being cultivated gradually, accepts the degree to the video charge form also higher and higher.

According to the 46th Statistical Report on China's Internet Development, by June 2020, China's number of online video users had reached 880 million, up 37.77 million from the end of March 2020, accounting for $94.5 \%$ of the total Internet users [1]. At the same time, users' awareness of paying for watching videos has improved. According to the survey of China's Internet entertainment market by IResearch in the first half of 2020, user payment has become the most important part of the revenue of video websites in China's online video industry, accounting for more than $50 \%$ [2].

In 2014, with the continuous maturity of $4 \mathrm{G}$ technology and the expansion of users, the users of Chinese video websites also showed a large-scale growth. At the same time, the market of Chinese video websites is gradually becoming saturated. To enhance market competitiveness, some large video websites begin to choose the mode of merger and reorganization or strong combination. After recombination and union, after several years of intense market competition, the basic 
pattern of Chinese video websites has been formed. As of the end of 2019, the overall user penetration rate of iQIYI, Youku, and Tencent's three video platforms was $80.2 \%$, while Mango TV and Bilibili were 9.2\%, ranking second. Sohu Video, PP Video, and Migu Video are in the third tier, with an overall user penetration rate of $6.7 \%$ [3].

\subsection{Literature review}

Chen found that network video media platform has a typical bilateral market structure. There are obvious cross-network externalities between bilateral users, and the cross-network externalities in bilateral users play an indirect role through network video media platforms. Regarding user payment, when the content differentiation degree is low, the pricing strategy of online video media platform users' unilateral payment is related to the content differentiation degree between platforms. In terms of advertising payment, platform enterprises will increase the number of undifferentiated ads or decrease the number of aversive ads when the proportion of aversive ads is high. After analyzing the cases of iQIYI, Tencent Video, and Youku, he found that the three platform enterprises all adopted the pricing strategy of bilateral users' cross-complementarity for the video content with advertisements, and the pricing of the three platform enterprises was the same. In terms of future user payment development, platform enterprises will shift from focusing on improving the differentiation between platforms to improving the quality of video content [4]. $\mathrm{Lu}$ proposed the development status of the iQIYI education channel and pointed out the problems in the marketing strategies of online education video websites, including serious content homogenization, uneven product quality, low user interaction rate, difficult promotion of user-paid courses, and constant disputes over copyright infringement. Based on the 4P theory, this paper analyzes the marketing mix strategy of the iQIYI education channel from four aspects: product, price, channel, and promotion. He thinks the iQIYI education channel's marketing strategy is still successful, providing inspiration and reference for other online education video websites to develop marketing strategies [5]. Based on 4P marketing theory, Dai studies the marketing strategy of Tencent video. It finds that the rapid development of Tencent video is inseparable from the positive marketing of the product, channel, promotion, and price. Firstly, it introduces the types of Tencent video products. Secondly, it points out that Tencent video covers the whole network and high-quality resources cater to the audience. The network broadcast exclusive copyright to attract users to make content, to create personalized products, Direct vertical network variety blank spots and other product strategies. The first is the price strategy of multiple price suits, and the second is the multiple price strategy for advertisers. Channel strategy is mainly based on WeChat, QQ social integration marketing; Precise marketing based on big social data; Tencent video whole product mobile terminal linkage marketing [6]. Aiming to monopolize the ride-hailing services market, Yang, Zhao, and Dai considered the different types of ride-hailing services, namely private car service and ride sharing service, and constructed a pricing decision model involving ride-hailing drivers and ride-hailing platforms. The Solution model of the Stankelberg game is adopted. The ride-hailing platform as the leader decides the service price, and the driver as the follower decides the optimal service time to provide. The research results show that the optimal service price decision of the platform is related to the commission rate, and the platform should pay attention to the scope of the total amount of time that the ride-hailing drivers can provide service. The service price difference between the private car mode and the free ride mode increases with the private car service cost, and the commission rate of the following windmill service decreases. At the same time, they discussed how the platform could incentivize drivers to provide more service when demand for service surges [7].

\subsection{Research framework}

This paper will analyze the specific pricing strategies of iQIYI and Tencent platforms. The reason for choosing this topic is that the author is also a video platform consumer and hesitating about which one is more costeffective among all video platforms. This study is interested in TV series, variety shows, pricing, and advertising marketing on video platforms.

Firstly, this paper will analyze the development status and trend of China's video website industry, especially the pricing strategy analysis. Secondly, this essay will also criticize the pricing strategy of some Chinese video websites. And finally, put forward a solution. To be more specific, optimizing the price strategy of the video website platform.

\section{METHODS}

This paper analyzes the pricing strategies of video network platforms by literature statistical analysis, case study and comparative analysis.

\subsection{Literature analysis method}

This study uses secondary data from journals, papers, industry reports, and websites for analysis and case studies, which is more typical and authoritative. In addition, the literature review was mentioned previously to help the author know more about the Chinese video website market. Also, this paper references some Industrial reports to analyze the development status and trend of the Chinese video website industry more efficiently. 


\subsection{Case study}

This paper chooses iQIYI and Tencent Video, which are two major Chinese video network platforms. It queries the data background of the above top 10 platforms from authoritative institutions to analyze and compare the pricing strategies of these two platforms better. The literature review found that iQIYI and Tencent Video have many similarities in their repricing strategies, and there is also a competitive relationship. As the two giants of Chinese video website platforms, these platforms can be studied very typically.

\subsection{Comparative analysis}

This paper compares the pricing strategies of iQIYI and Tencent Video, two major video network platforms. Comparing these two video platforms can make us more clearly understand the differences in pricing strategies and the ingenuity in the application. The competition between the two platforms can also raise readers' interest points.

\section{RESULT}

\subsection{Development status and trend analysis of Chinese video website industry}

From 2014 to 2017, the market size of China's video website industry has grown from 24.9 billion yuan to 95.2 billion yuan. The video website industry is developing very rapidly, and it is expected that the market size of the video website industry will continue to expand. In 20172020 , the overall compound growth rate will reach $19.1 \%$, and in 2010, the market size of the entire video website is expected to increase to 191.6 billion yuan [8].

The war between video websites in China has been going on for many years. In terms of the current situation, the representative websites are iQIYI, Youku, and Tencent, and the competition has always been quite fierce. The battle for high-quality video content is becoming increasingly fierce to compete for members and user flow and get rid of losses. However, each video website has a huge user base and data traffic, but still unable to profit, unable to get rid of the situation of trapped loss. At present, the main source of income of the network video market is still advertising income and member income. In recent years, the membership scale of Chinese mainstream video websites continues to expand at a gratifying rate, and the member income and advertising income continue to grow.

\subsubsection{Overview of Chinese video website industry}

2018 is a year of explosive growth of paid membership model for video websites. According to iQIYI's 2018 Q4 financial report, the total revenue of iQIYI reached 25 billion yuan, the scale of subscription members reached 87.4 million, and paid members accounted for $98.5 \%$. iQIYI added 36.6 million net subscription members in the whole year, with the number of subscription members increasing by $72 \%$ year on year [9]. The expansion of paid membership has made iQIYI's membership revenue overtake advertising revenue for the first time to become the most important source of revenue. Full-year membership revenues were Rmb10.6bn, while advertising revenues were Rmb9.3bn. Tencent Video's membership reached 82 million in the third quarter of 2018, on a par with iQIYI's. However, the increase of membership income will affect the traditional advertising income to a certain extent. The membership model realises the users' paying way to watch the online video content in advance, download the high-definition and ultra-high-definition online video, and avoid watching the advertisements in the video content.

\subsubsection{Consumer analysis}

In China, most users do not have a strong awareness of paying for watching videos, and video websites must improve the existing video open mode to make profits. Hence, users are willing to pay to watch videos. In the current situation that almost the entire video industry is not profitable, it is no longer realistic to rely on large investments to buy drama and fight for copyright to grab users. Moreover, the free content of video websites can bring traffic growth, but if the traffic is not well realized to drive revenue, it will make the investors behind video websites very passive. To make the paid content of the website have better development, we need to overturn the traditional TV station to rely on advertising to obtain revenue thinking and further improve the video revenue model. It is worth noting that with the strengthening of the protection of knowledge products and the gradual improvement of users' awareness of knowledge payment, content payment accounts for an increasing proportion of the total income of video websites. Data show that in 2018 , the content payment revenue of video platforms reached 64.559 billion yuan, accounting for more than 30 per cent of the total revenue [10].

\subsubsection{Brand analysis of Chinese video network platform}

In 2005, video websites began to sprout in China. The public is familiar with video websites such as iQIYI, Youku, Tudou, and Tencent Video. Among foreign video websites, YouTube is developing rapidly. Internet video websites are no longer limited to providing single video service content in the continuous integration and development of the pan-entertainment industry. The industrial chain is gradually extending to peripheral services and comprehensive entertainment services.

2018 has been an active year for capital flows in the Internet video industry, with iQIYI listing on NASDAQ in February and Bilibili's shares on the same stock 
exchange in March. In addition, although unlisted, there are many Internet video enterprises with strong financial support. For example, Alibaba owns $18.3 \%$ of Youku Tudou, one of the video broadcasting giants, and Tencent has also established a wholly-owned video broadcasting platform. In the capital market, listing and $m \&$ a activities of Internet video websites will also become more frequent. According to the CSMAR database, during the five years from 2015 to 2020 , more than 1,400 capital transactions such as major asset reorganization, listing and $\mathrm{m} \& \mathrm{a}$ of Internet enterprises doubled the number of transaction events compared with the five years during 2010 and 2015 [11]

\subsection{Current situation of pricing strategy of Chinese video website}

From the birth of China's first video website in 2006 to today, the development of the Chinese video website has been less than ten years, but its development in a short time is amazing. Video websites rely on the Internet, mobile phones, and other new media. In a short period, it has been a qualitative improvement.

Since 2015 , driven by many factors, the number of paying users of video websites has increased rapidly, and the proportion of member income in the overall income has increased. By December 2015, the number of online payment users reached 416 million, with a growth rate of 36.8 per cent. Among them, mobile online payment users reached 358 million, with a growth rate of $64.5 \%$ [12]. Thus, the development of online video in China has entered the era of payment, and the online video media platform is gradually getting rid of the dependence on advertising revenue [13].

First of all, the open content and mode provide the vast number of Internet users with the opportunity to upload and publicly share their videos. As a result, usermade videos have almost become the main content of significant video websites. Secondly, the integration of traditional media and new media; Video websites and TV, broadcast, press, and other traditional media cooperation, is the most significant feature.

\subsubsection{Pricing strategy analysis of $i Q I Y I$}

iQIYI currently has three main profit channels: advertising revenue, membership payment, and selfproduced programs. In recent years, advertising has fallen as a percentage of total revenue but remains a key source of profits for iQIYI. Therefore, it is necessary to continuously produce high-quality marketing products and optimize precise delivery procedures to attract advertisers. The proportion of member payments is rising. According to the financial report at the end of the third quarter of 2020, iQIYI's member service revenue is 4 billion yuan, up 7\% year on year, accounting for more than half of the total turnover during the period. Member payment business has become the most important revenue-increasing engine of $\mathrm{iQIYI}$.

iQIYI's payment business model is mainly divided into two parts. The first part is the pay-per-view mode of network movies. The paid online movie model means that the producer and distributor of video share the revenue from pay-per-view video according to the agreed quota. For example, in iQIYI pay-per-view movie business, network movie on-demand price is 5 yuan, each film free and proved for 5 minutes, more than 5 minutes platform will regard it as an effective click broadcast, and video production can gross from every time the effective demand, iQIYI platform can get the rest of the income.

The second part is the membership system, the main part of iQIYI's payment business. iQIYI's high-quality user experience is an important factor in promoting the conversion of free users to VIP members. iQIYI's paid products are mainly divided into two categories. One is the free film library for members, which provides highly popular content. In addition, paying members can enjoy the benefits of content privilege, identity privilege, function privilege and so on, specifically including skipping commercials, massive movie library, super network drama and live idol exclusive activities, birthday gift packages, special movie tickets, and other exclusive benefits.

iQIYI's ad sales service provides advertisers with various choices, including new forms of advertising such as product placement and ad customization and the most traditional patch advertising, pause advertising, and so on.

With the development of mobile communication devices, more users watch videos more frequently on mobile terminals than on PC terminals. However, the advertising ratio of mobile phone terminals is far lower than that of PC terminals because the mobile terminal has not established an effective media monitoring method, and it is more convenient to measure the advertising effect on PC terminals. iQIYI in 2014, to solve this contradiction, set up in the device and the iPhone video advertising monitoring system, the traditional PC used widely in advertising sales mode into advertising monitoring on the mobile side, in the video website between advertisers advertised on mobile devices and established a relatively complete solution, and set up a scientific and effective evaluation system for cross-media advertising is proposed. iQIYI's innovation in advertising charging mode effectively avoids the problem of insufficient objectivity of mobile data and increases the confidence and enthusiasm of advertisers to advertise on video website platforms.

\subsubsection{Tencent video pricing strategy analysis}

Tencent's video pricing strategy implements diversified pricing. For this reason, it classifies the member users on the video website. In addition to regular 
users, there are also super membership and membership, who have different pricing and enjoy different functions, products, and services.

Tencent video is based on differentiated pricing to meet the different needs of consumers. Its membership system is divided into two kinds: one is a super member; The other category is membership. For example, ordinary members can watch videos on mobile phones, computers, and ipads. Membership fees are paid monthly, quarterly, or annually, but they cannot watch videos on TV. At the same time, the system will remind users of the remaining time of membership benefits and recommend that users make up the difference to upgrade to super membership.

In addition, there are many advertisers on the Tencent Video website to users, and the pricing of these customers is also flexible. In recent years, Tencent video's main revenue has come from advertising. Compared with traditional advertising, the cost of advertising on video websites is relatively low. In addition to the advantages of advertising on a comprehensive video website, there are more formats, which are more suitable and can meet the market effect. In the face of advertisers' pricing strategies, they need to consider various influencing factors. For example, they must consider product production, optimization and brand image creation, different advertising prices, and other aspects.

In sum, as shown in Table 1, all major online video media platforms have the same charging mode for providing advertising-free video services. As a result, consumers' services on major online video websites mainly watch videos without advertising and watching VIP videos. As a result, membership income is highly dependent on the video site's library of films and the popularity of new films.

Table 1. Online video platform user payment data

\begin{tabular}{|c|c|c|c|c|}
\hline $\begin{array}{l}\text { The } \\
\text { platfo } \\
\text { rm }\end{array}$ & \multicolumn{2}{|l|}{ iQIYI } & \multicolumn{2}{|c|}{ Tencent video } \\
\hline $\begin{array}{l}\text { Mem } \\
\text { ber } \\
\text { name }\end{array}$ & $\begin{array}{l}\text { Golden } \\
\text { members } \\
\text { hip }\end{array}$ & $\begin{array}{l}\text { Star } \\
\text { members } \\
\text { hip }\end{array}$ & $\begin{array}{l}\text { Tencent } \\
\text { video } \\
\text { members } \\
\text { hip }\end{array}$ & $\begin{array}{l}\text { Super } \\
\text { members } \\
\text { hip }\end{array}$ \\
\hline $\begin{array}{l}\text { Servic } \\
\text { e } \\
\text { featur } \\
\text { es }\end{array}$ & $\begin{array}{l}\text { No } \\
\text { commerci } \\
\text { als, free } \\
\text { viewing. }\end{array}$ & $\begin{array}{l}\text { No } \\
\text { commerci } \\
\text { als } \\
\text { advance } \\
\text { viewing, } \\
\text { free } \\
\text { viewing, } \\
\text { five clients }\end{array}$ & $\begin{array}{l}\text { No } \\
\text { commerc } \\
\text { ials, free } \\
\text { viewing. }\end{array}$ & $\begin{array}{l}\text { No } \\
\text { commerc } \\
\text { ials, } \\
\text { clearer } \\
\text { image } \\
\text { quality, } \\
\text { advanced } \\
\text { viewing }\end{array}$ \\
\hline
\end{tabular}

\begin{tabular}{|l|l|l|l|l|}
\hline $\begin{array}{l}1 \\
\text { mont } \\
\mathrm{h}\end{array}$ & 25 yuan & 60 yuan & 30 yuan & 50 yuan \\
\hline $\begin{array}{l}\text { same time } \\
\text { mont } \\
\mathrm{h}\end{array}$ & 68 yuan & 138 yuan & 68 yuan & 98 yuan \\
\hline $\begin{array}{l}12 \\
\text { mont } \\
\text { h }\end{array}$ & 248 yuan & 418 yuan & 253 yuan & 288 yuan \\
\hline
\end{tabular}

\section{DISCUSSION}

\subsection{Problems existing in the pricing strategy of Chinese video websites}

Although the pricing strategy of Online video platforms in China has been relatively perfect, there are still some problems and areas to be improved.

\subsubsection{The problem of commercial breaks}

For the free mode of advertising, video sites will usually be placed at the beginning of the video, and the actual effect may not be good. Some viewers do not watch commercials after they know the time of commercials but watch videos after commercials. Therefore, this paper can investigate and analyze users' viewing habits to determine the reasonable way of advertising breaks and the corresponding advertising time. For example, it is better to watch a video with several short breaks.

\subsubsection{Video resolution}

This study can add other operating costs of video sites to the model. Video sites are also difficult to profit from because of the huge cost of user bandwidth. If video websites provide high-definition and smooth videos to users, they need to pay high bandwidth costs. If a video site provides low-resolution videos to its users, it can save bandwidth costs, but users will have a lower opinion of the video site. Therefore, video sites need to determine what kind of clear degree of open video.

\subsection{Optimization of video website platform pricing Strategy}

Combined with the theoretical analysis and case analysis mentioned above, this section points out the pricing strategy of video website platform operators. In terms of user fees, operators should be committed to expanding users' demand for payment, optimizing 
pricing strategies, and optimizing the payment services provided by the platform to enhance the additional utility of members. In terms of advertising fees, platform operators need to reduce their dependence on advertising business and constantly innovate advertising business forms.

\subsubsection{Innovate advertising content and form}

With the continuous development of the video website industry, the user's rebellious psychology to advertisement is intense. Therefore, on the one hand, video website platform operators should reduce the dependence of platform operation on the advertising business, create more profit sources and optimize the advertising pricing model. On the other hand, platform operators need to optimize and innovate the form of advertising business constantly. At present, the users of Chinese video websites are constantly migrating to mobile terminals. Therefore, the platform operators of video websites can establish an effective advertising charging mode on mobile terminals to attract advertisers to increase their advertising investment on mobile terminals.

\subsubsection{Video website platform operators should publicize the concept of payment and cultivate users' payment habits.}

The previous analysis has pointed out that the payment business is the key for Chinese video website platform operators to profitability. In recent years, the user scale of China's video websites has kept growing, and the proportion of paying users is not high, and the growth rate is relatively low. It can be seen that there is a long way to go to expand the scale of paying users. Platform operators need to cultivate users' awareness of paying for membership services. Paid memberships are themselves an emerging service that can be marketed and packaged as a special good. Major video website platform operators can visually promote the invisible paid membership system by publicizing the concept of payment, changing the fixed thinking of current video website users, and cultivating their payment habits.

\subsubsection{Premium content}

Platform operators need to focus on developing personalized membership privileges. Current video website platform between the content of the increasing level of differentiation, the rich, high-quality copyright content library, the members to pay membership privileges as a video website platform and attract advertisers to advertise, the basis of quality such as copyright, boutique self-control not only can effectively help users incremental, also can through the accumulation of a large number of users, more efficiently and accurately match the user demand, creation produce better content. At the same time, in terms of enhancing the additional utility of paid members, it attracts users to watch various video content such as movies, homemade dramas, and variety shows.

\section{CONCLUSION}

In recent years, the paid membership model of video websites has been growing rapidly. As a result, video websites need to improve their existing video revenue model. iQIYI and Tencent both have three main profit channels: advertising, membership, and self-made variety shows. As more and more users like to watch videos on mobile clients, iQIYI has widely applied the traditional PC advertising sales model to mobile client advertising monitoring. On the other hand, Tencent Video has set up tips on its platform to stimulate consumption, and it classifies its video site's member users for targeted services. Moreover, this paper also puts forward the problems in Chinese video network platforms, such as advertisement placement and video definition, and makes targeted suggestions for their optimization.

This paper can help more platform users choose video platforms and enable video platforms to optimize and correct their shortcomings in their strategies to promote the benign development of video website pricing strategy and provide similar pricing strategy reference for other Internet enterprises mainly providing content services. Moreover, this study helps us to have an in-depth understanding of the status quo and a series of problems arising from the development of video websites. Finally, it provides some suggestions and strategies for solving the challenges faced by the development of video websites, which can serve as valuable information and basic background for the pricing strategy of video websites.

Although the research of this paper has gained some research results, there are still many problems in the network video industry that deserve further exploration. In the research process of this paper, the author found that problems worthy of further study in the online video media industry are embodied in the following aspects. First, secondary data is mainly used in this paper, but the primary data is lacking. Secondly, with the rapid development of modern short videos and live videos, the author can also study its profit model and pricing strategy.

In the future, the author can obtain more authentic and reliable primary data through a survey, interview, and other methods

\section{REFERENCES}

[1] The 46th Statistical Report on Internet Development in China, China Internet Network Information Center (CNNIC) 
[2] 2020Q1-2020Q2 China Internet entertainment Market Data Report, IResearch consulting, 2020

[3] 2019 Chinese Network Audio-visual Development Research [EB/OL]. Sohu, 2019-05-27. DOI: https://www.sohu.com/a/316802357_728306.

[4] Chen. Research on pricing Strategy of online video media Platform [D]. Zhejiang University of Finance and Economics, 2017.

[5] Lu. Research on marketing Strategy of iQIYI Education Channel Based on 4P Theory [D]. Hebei University, 2017.

[6] Dai. Research on Tencent video Marketing Strategy [D]. Xinjiang Universit, 2019.

[7] Yang, ZHAO and Xu. Research on Service Pricing Strategy of Private Car and ride sharing with Platform Monopoly [J/OL]. Industrial Engineering and Management :1-11, 2021.

[8] China Internet Development Report (2021)

[9] iQIYI released 2018 financial results: annual revenue over 25 billion members 87.4 million U. TV Guide, 2019.

[10] Zhu. Research on revenue optimization of video websites considering copyright types and charging modes [D]. University of Science and Technology of China, 2021.

[11] Wei. Research on the Value Evaluation of sharing video websites from the perspective of user value [D]. Yunnan University of Finance and Economics, 2021.

[12] The 38th Statistical Report on Internet Development in China.

[13] Chen. Research on pricing Strategy of online video media Platform [D]. Zhejiang University of Finance and Economics, 2017. 